\title{
Evaluation of microdamage initiation in Z-pinned laminates by means of automated RVE computations
}

\author{
Gerrit Pierreux ${ }^{\mathrm{a}, \mathrm{b}, *}$, Ling $\mathrm{Wu}^{\mathrm{c}}$, Danny Van Hemelrijck ${ }^{\mathrm{a}}$, Thierry J.Massart ${ }^{\mathrm{b}}$ \\ ${ }^{a}$ Vrije Universiteit Brussel, Department of Mechanics of Materials and Constructions, \\ Pleinlaan 2, 1050 Brussels, Belgium \\ ${ }^{b}$ Universite Libre de Bruxelles, Building, Architecture and Town Planning Dept., Avenue \\ Fr. Roosevelt 50, CP 194/02, 1050 Brussels, Belgium \\ ${ }^{c}$ University of Liège, Computational and Multiscale Mechanics of Materials, Chemin des \\ Chevreuils 1, B-4000 Liège, Belgium
}

\begin{abstract}
Z-pinning was originally designed to improve the delamination toughness and the impact resistance of composite laminates. However, there is extensive experimental evidence that this improvement is accompanied by a reduction of the in-plane properties. The main mechanisms responsible for this deterioration are the local change in fiber content, fiber distortion, and the inclusion of resinrich regions near the Z-pin. The shape of these geometrical features strongly depends on the laminate stacking sequence and on pin parameters such as pin diameter, pin content, and initial pin inclination angle. Their shape complexity challenges analytical modelling approaches which are currently used to generate RVE geometries for simulations.

A computational approach is presented to generate such geometrical models. Resin-rich regions are modelled by initially straight discretized lines which are gradually shaped by a set of geometrical operations mimicking pin insertion, pin rotation and fiber deflection. Fiber distortion is modelled in a post-processing stage in cross-sections accounting for the preservation of the amount of fibers. These models are then transformed into finite element mechanical models in order to investigate how local fiber volume fraction changes, fiber misalignment,
\end{abstract}

\footnotetext{
${ }^{*}$ Corresponding author

E-mail address: gerrit.pierreux@gmail.com (G. Pierreux).
} 
or distortions in reinforcement due to pin rotation, affect the global stiffness and local stress concentrations.

Keywords: Z-pinning, internal geometry, mechanical properties, multiscale modelling

\section{Introduction}

Z-pins are used in the through-thickness direction of laminates to increase their delamination resistance [17]. Various methods have been proposed for inserting such pins. The most widespread technique, used both in industry and research, consists of pushing the pins from a foam bed into the laminate by applying a pressure combined with ultrasonic vibration [8, 9. The part of the pins not embedded in the laminate is removed afterwards by chamfering, followed by a further consolidation and curing process in an autoclave (see Fig 1 1 $)$. Apart from the intended increase in delamination resistance, experimental results have shown a reduction of the in-plane mechanical properties, with an extent dependent on pin parameters such as their spacing, diameter and insertion pattern [10 14. The reduction is caused by reinforcement distortions, which take place during pin insertion and further processing, and can be characterized by resin-rich regions and fiber distortions near the pin 14 17. The effect of these geometrical features on the in-plane mechanical properties needs to be understood in order to optimize the design. A computational homogenization approach that uses a periodic mesoscopic unit-cell model has shown positive results in understanding and even predicting the mechanical behavior of fiber-reinforced composites with other geometrical arrangements [18 26]. Such an approach requires however the generation of mesoscopic geometrical models that include the main geometrical features affecting the in-plane mechanical behavior.

Resin-rich regions and fiber distortions or reorientations around the pin are gradually shaped during pin-insertion (during which the fibers are pushed aside) 
and further processing (during which the pin rotates), as illustrated in Fig. 17. A small pin content results in eyelet-shaped regions, Fig. 1 b, while larger pin contents result in channel-shaped resin-rich regions that form due to distortions from neighboring pins. Upon further processing, pin rotation can take place, whereby the fibers are pushed more to one side of the resin-rich region, which results in distortion of its symmetrical shape (see Fig. 11.). The extent of these asymmetrical distortions in a lamina depends on the vertical position of the lamina relative to the pin rotation center, and on the main fiber direction in the lamina relative to the direction of pin rotation. The fiber-reinforced distorted zone around a pin is mostly assumed to be rectangular with a width related to the pin-diameter. Experimental data on variations in local fiber volume fraction and local fiber direction inside this region are, to the best knowledge of the authors, are rather scarce in the literature. Coupled with these issues, an outof-plane component, known as fiber crimp, can also arise during pin-insertion and pin-rotation. Furthermore, swelling of the laminate, fiber-breakage, and fiber-weaving in irregular pin patterns (see Fig. 11d), upon pin insertion, and interfacial cracking between the pins and composites caused by thermal shrinkage of the pin after curing, can also be present. All these geometrical features can alter the local stress distribution and consequently need to be included in the RVE geometries used in simulations.

In literature, an analytical approach was developed to include these geometrical features in a mesoscopic geometrical model [27 29]. In these contributions, symmetrical eyelet-shaped resin-rich regions are represented either by a cosine shape, an orthorhombic shape, or a diamond-shape, whereas for channel-shaped regions a rectangular shape is used. The fiber distorted zone is mostly represented as a rectangular area around the pin. Local fiber misalignment reduces linearly from a maximum at the resin-rich region boundary to zero outside the fiber distorted area (see Fig. 17). The local fiber volume fraction is mostly assumed constant in each lamina. However, no existing model can provide the asymmetrical shape of a resin-rich region due to pin rotation or can ensure a 
seamless transition between eyelet- and channel shaped resin-rich regions.

Therefore, in the present contribution, a computational approach capable of generating geometries for all of these geometrical features in laminates is presented. Resin-rich regions are represented by discretized lines, which are initially straight and are then gradually shaped using a set of geometrical operations (see Fig. 2), with concepts similar to [30, 31. The fiber-reinforced distorted zone is obtained in a post- processing step from these deformed discretized lines and is modelled in cross-sections perpendicular to the main fiber direction of the considered lamina. Preservation of the amount of fibers in a cross-section is used to calculate the model parameters. The generated RVE geometrical model is then transformed into a finite element model in order to investigate to what extent geometrical features affect the global stiffness and local stress distributions within laminates given a prescribed stacking sequence.

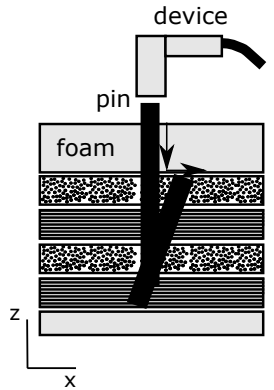

a)
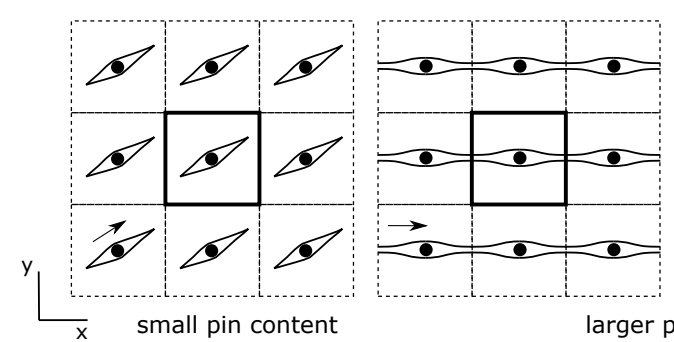

larger pin content

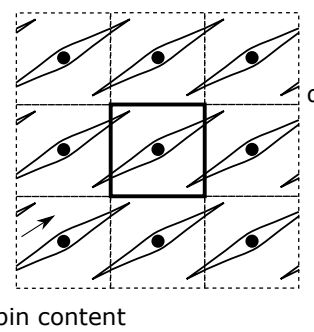

b)

Figure 1: Z-pinned laminates: (a) pin insertion and pin chamfering, (b) eyelet- and channelshaped resin-rich regions for small and large pin content, (c) asymmetrical-shaped resin-rich region and fiber-reinforced distorted zone (due to pin rotation), (d) fiber weaving (due pin pattern imperfections) and (e) local fiber-reinforced distorted zones near the pin.

\section{Geometrical model generation}

Unit-cell models with a single which can be inserted vertically or initially inclined are generated. A regular pin pattern, a mesoscopic initially homogeneous 
fiber volume fraction (before pin insertion) and fiber direction in each undistorted lamina, and a straight and rigid pin are hereby assumed. Fiber-breakage, localised through-thickness crimping of the in-plane fibers, and interfacial cracking between pins composite are not considered in the modelling.

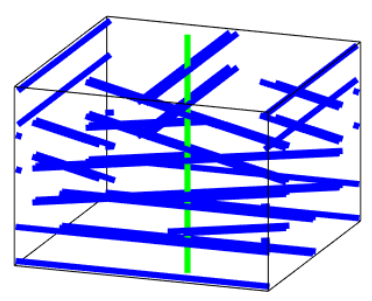

1)

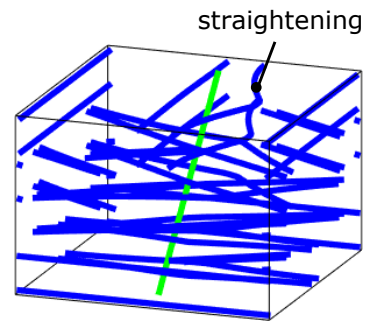

4)

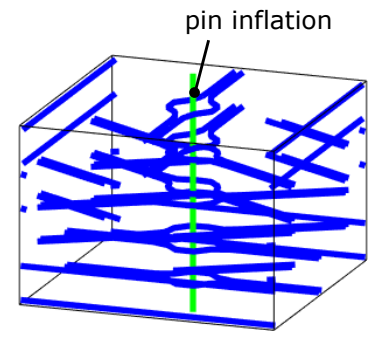

2)
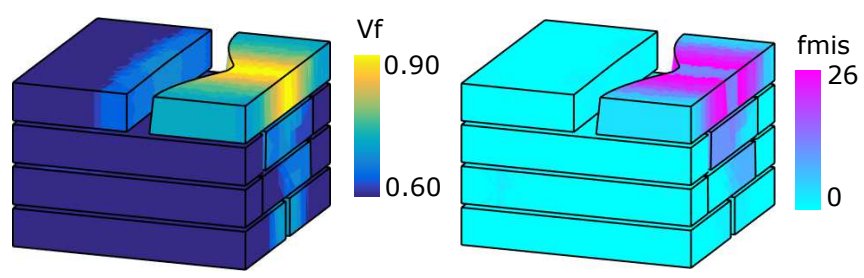

5)

Figure 2: Step-wise generation process: (1) initial model, (2) pin inflation, (3) pin rotation, (4) lamina straightening, (5) fiber volume fraction $\left(V_{f}\right)$ and fiber misalignment $\left(\theta_{\text {mis }}\right)$ distributions in a post-processing step.

\subsection{Initial model}

The initial model accounts for potential resin-rich regions, which are caused by pin insertion, and the presence of an initially inclined pin. Lines are used to represent these geometrical features. They are discretized and a line radius is assigned to enable the shaping of the fibers and the detection of interpenetrations between the inserted pin and the reinforcement.

The pin is positioned at the center of the unit-cell and can be initially inclined. A single line is used to represent the pin. Its position is determined 
by a rotation center, an angle of inclination plane and an initial pin inclination angle, as illustrated in Fig. 3. The line radius is taken as the pin radius and the line representing the pin is not further discretized.

The resin-rich regions in a lamina are aligned with the main fiber direction $\vec{f}_{\text {lam }}$. They originate either from the insertion of a pin within the unit-cell or from neighboring pins for larger pin contents in lamina with fibers which are not aligned with one of the pin pattern directions. Straight lines are used to represent the boundaries of resin-rich regions. These lines are aligned to the main fiber direction of the considered lamina and are positioned through-itsthickness at both sides of potential opening locations. The position of the lines for both a $0^{\circ}$-lamina and $30^{\circ}$-lamina is illustrated in Fig. 3 . For the $30^{\circ}$ lamina, the presence of potential resin-rich regions caused by pins belonging to neighboring unit-cells is accounted for. The positioning of lines (see Fig. 3b) can easily be achieved by considering a cross-section that is perpendicular to the main fiber direction. For inclined pins, the lines at the top and bottom of a lamina are displaced. The lines are uniformly discretized into line segments described by nodes (not to be confused with FE discretisation nodes) with a length equal to one third of a standard pin diameter $(280 \mu \mathrm{m})$.

\subsection{Geometrical shaping operations}

The discretized lines of the initial model are next shaped by a set of geometrical operations that are applied sequentially and mimic the pin and fiber kinematics taking place during the manufacturing process (see Fig. 2). For instance, the pin insertion is mimicked by an inflation operation applied on an embedded pin. The chamfering process applied in order to remove the excess length of the pins after insertion and the transverse compaction during laminate consolidation as part of the cure process causes the pin rotation which is represented geometrically by a rotation operation. The locally deformed shape of the lines near the pin are smoothed by a straightening operation. Upon inflation, the interaction between the fibers and the pin is accounted for by a contact 

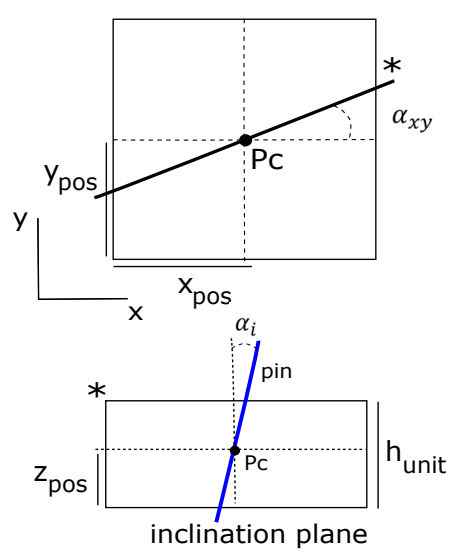

a)
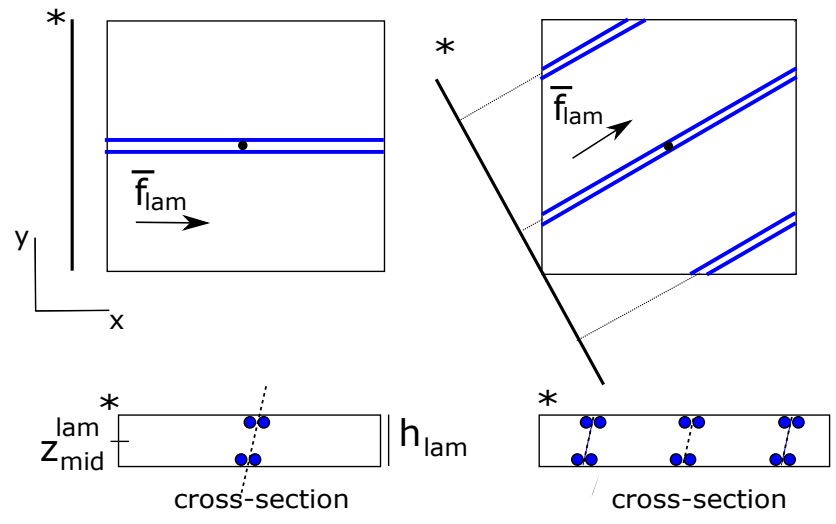

b)

Figure 3: Initial model: (a) pin, (b) lamina with fiber aligned and not aligned with a pin pattern direction (left and right respectively).

treatment. Furthermore, the inflation and rotation operations are applied incrementally to prevent lines from crossing over each other before interpenetration detected by the contact treatment. The nodes used in the line description can then be constrained to the lamina boundaries in order to facilitate an easier control of the final geometrical model. The geometrical operations are governed by the following equations.

\subsubsection{Inflation operation}

The pin inflation operation incrementally increases a line radius $R_{\text {line }}$ from zero to a final radius:

$$
\begin{aligned}
& R_{\text {line }}^{n}=R_{\text {line }}^{n-1}+\frac{R_{\text {line }}}{n_{\text {infl }}} \\
& R_{\text {line }}^{0}=0 \text { and } n: 1 \rightarrow n_{\text {infl }}
\end{aligned}
$$

where $n_{\text {infl }}$ is the number of inflation increments.

\subsubsection{Rotation operation}

The rotation operation rotates a line in a certain inclination plane, around a rotation center, and with an inclination angle $\alpha_{n}$ that is incrementally increased 
from $\alpha_{i}$ to a final value $\alpha_{f}$ :

$$
\begin{aligned}
& \alpha^{n}=\alpha^{n-1}+\left(\frac{\left(\alpha_{f}-\alpha_{i}\right)}{n_{\text {rot }}}\right) \\
& \alpha^{0}=\alpha^{i} \text { and } n: 1 \rightarrow n_{\text {rot }}
\end{aligned}
$$

where $n_{\text {rot }}$ is the number of rotation increments.

\subsubsection{Straightening operation}

The straightening operation repositions the nodes of a line after local displacements induced by inflation or a rotation of the pin. Each node $i$ of a line is moved to the middle of the segment connecting the neighboring nodes (see Eq. 3 and Fig. 4 2). This operation mimics the fact that a fiber under tension

moves transversally with the largest movement taking place in regions with the highest curvature.

$$
\vec{x}_{i}=(1 / 2) \cdot\left(\vec{x}_{i-1}+\vec{x}_{i+1}\right)
$$

This operation is applied to shape the lines near the pin of the unit-cell and is extended to account for the effect of potential resin-rich regions from neighboring unit-cells on the geometry of the considered unit-cell. Before applying the straightening operation, the lines near the unit-cell pin (master lines) are extended, as illustrated in Fig. [4 for both channel-shaped ( $0^{\circ}$-lamina) and eyelet-shaped ( $30^{\circ}$-lamina) resin-rich regions. After straightening, the extended part can be removed ( $0^{\circ}$-lamina) or used to shape the additional eyelet-shaped regions (30 -lamina). By applying this operation a number of times, a desired length of a resin-rich region can be generated.

\subsubsection{Contact treatment}

The contact treatment detects interpenetrations locally between two discretized lines, based on their attributed radius and by evaluating the distance between a node on a line and all other lines. When interpenetration is detected, the concerned nodes are moved in the opposite direction as illustrated by Fig. [5] 


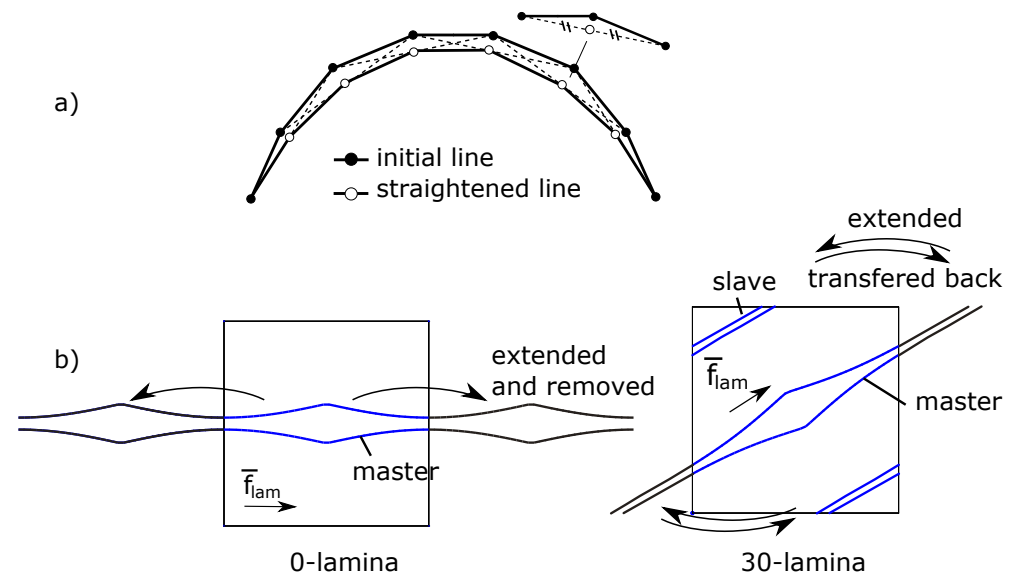

Figure 4: Straightening operation: (a) concept inspired from [30, (b) periodic line straightening.

$$
\begin{aligned}
& d_{\mathrm{int}}^{P}=\min \left[\left(d^{P}-\left(R_{1}+R_{2}\right)\right), 0\right] \\
& \vec{x}_{\mathrm{mov}}^{P}=\alpha \cdot d_{\mathrm{int}}^{P} \cdot \vec{D}_{P}
\end{aligned}
$$

where $d_{i n t}^{P}$ is the distance between lines at node $\mathrm{P}$, and $R_{1}$ and $R_{2}$ are the line radii. The control of the movement of node $\mathrm{P}, \vec{x}_{\text {mov }}^{P}$, is defined by Eq. $4 \mathrm{~b}$ in an interpenetration resolving step that uses the normal from the node on the pin, $\vec{D}_{P}$, multiplied by a fraction, $\alpha$, and the local interpenetration distance or the overlap between both lines. As the pin is not discretized and is considered to be rigid, only the nodes belonging to the boundaries of the resin-rich region are moved such that dissimilar lines are not interpenetrated.

\subsubsection{Boundary conditions}

The position of nodes can be constrained during generation by simply moving the nodes back to their desired location after each contact treatment. This procedure is implemented to constrain the nodes on the unit-cell border during generation, and to have a control over the bottom and top surface of a lamina in the final geometrical model. 


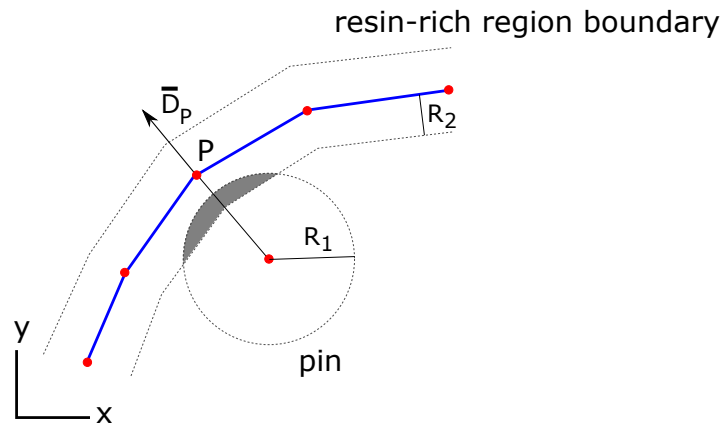

Figure 5: Contact treatment.

\subsection{Fiber-reinforced distorted zone modelling}

\subsubsection{Discretised lines into cross-sections}

The deformed lines, which represent the boundary of potential resin-rich regions, are used to create cross-sections representing the fiber-reinforced region. Additional lines are added to represent the in-plane borders of the lamina (or unit-cell) which are positioned on the in-plane borders and through-thethickness (see Fig. 6a). All the lines are then intersected by planes perpendicular to the main fiber direction that are defined at regular intervals along a diagonal of the unit-cell (see Fig. 6a). On each plane, a contour-line is generated around each set of intersection points that belong to a certain part (see Fig. 6p). Adjacent contour-lines for which the opening between them is less than an opening distance are then merged.

\subsubsection{Fiber volume fraction and fiber direction}

Fig. 7 a depicts an arbitrary cross-section at position $u$, with the origin of the local coordinate axis system positioned at the center of the pin and the $u$-axis aligned with the main fiber direction. The area within the considered crosssection, next to the resin-rich zone, is the area where the fibers are distorted due to pin insertion. The cross-section is deformed only at the sides adjacent

to the resin-rich region and the shape of the cross-section can be considered to be rectangular or trapezoidal, for a vertical or inclined pin respectively. A distorted zone with a width that is constant along the lamina thickness is assumed 


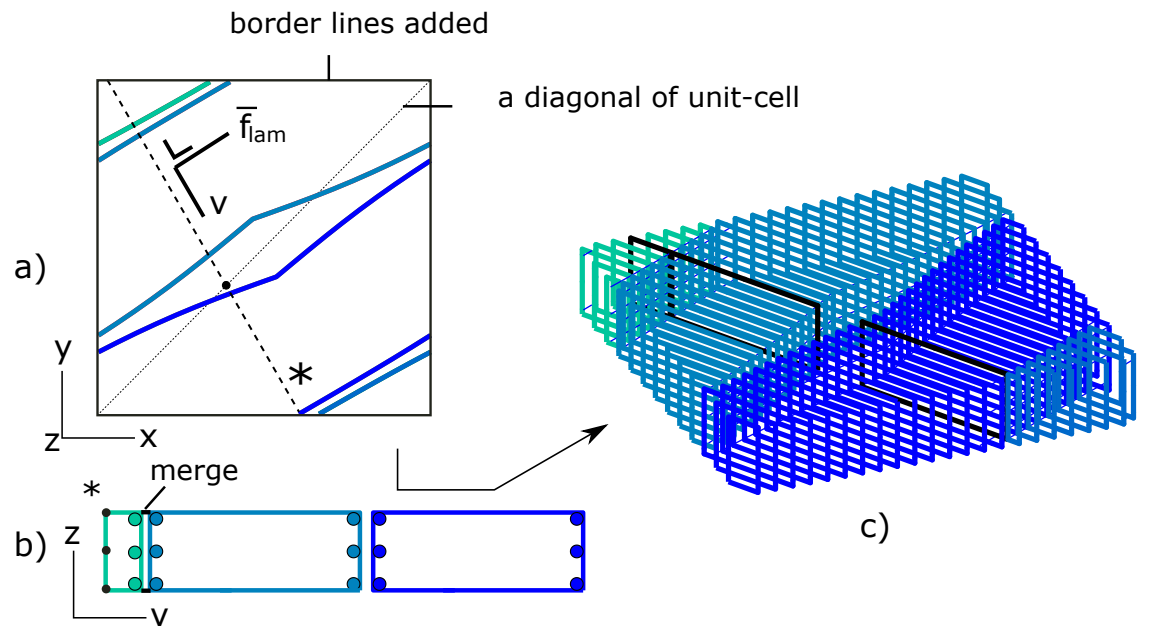

Figure 6: Contouring the discretised lines of a lamina with openings into cross-sections: (a) additional lines added on the in-plane lamina borders, (b) cross-sections generated around individual point set, (c) a 3D representation of the generated cross-sections.

and can be characterized by two regions with differing fiber volume fraction and fiber direction profiles, as illustrated in Fig. 7b. The first region, which is the closest to the resin-rich region, is characterized by a constant but increased fiber volume fraction $V_{f, c}$ and a constant fiber direction $\vec{f}_{l o c}$ that is identical to the local tangent at the boundary of the resin-rich region $\vec{f}_{\text {bound }}(u)$. The second region, which is located between the first region and the undistorted zone, is characterized by a fiber volume fraction and fiber direction that are linearly varying between the values in these two regions. The width of each region is represented respectively by $d_{1}(u)$ and $d_{2}(u)$. The fiber-reinforced distorted model in a cross-section at position $\mathrm{u}$ is then defined by the parameters $d_{1}(u)$, $d_{2}(u)$ and $V_{f, c}(u)$.

The value of these parameters is obtained by considering the preservation of the amount of fibers in the cross-section during the model generation. An amount of fibers equivalent to the area $A_{d f}(u)$ multiplied by the volume fraction, $V_{f, l a m}$, is redistributed locally in a distorted zone of the cross-section according 
to the assumed fiber volume fraction profile, which gives rise to the following relation:

$$
A_{\mathrm{df}}(u) \cdot V_{f, \mathrm{lam}}=\left(A_{d 1}(u)+\frac{A_{d 2}(u)}{2}\right) \cdot\left(V_{f, \mathrm{c}}(u)-V_{f, \text { lam }}\right)
$$

where the areas $A_{d f}(u), A_{d 1}(u)$ and $A_{d 2}(u)$ are illustrated in Fig. 7 . Since these areas can be considered rectangular or trapezoidal, Eq. (5) can be altered to define:

$$
v_{0}(u) \cdot V_{f, \operatorname{lam}}=d_{1}(u)+d_{2}(u) \cdot\left(V_{f, c}(u)-V_{f, \operatorname{lam}}\right)
$$

where $v_{0}(u)$ is the local position of the resin-rich region boundary.

For the parameters $d_{1}(u), d_{2}(u)$ and $V_{f, c}(u)$ additional assumptions are required to fully describe the fiber distribution in each cross-section. First, the distorted zone parameters $d_{1}(u)$ and $d_{2}(u)$ are assumed to be constant in each cross-section. Secondly, $d_{2}$ is considered a multiplication of $d_{1}$ :

$$
d_{2}=k \cdot d_{1}
$$

where $k$ is further called the distorted zone parameter and can be used to regulate the fiber-reinforced distorted zone width (see section 3.2). Thirdly, a value of $V_{f, c}(0)$ near the pin is assumed, which takes into account a maximum threshold to account for the fact that a maximum fiber volume fraction cannot be exceeded. These assumptions then allow the subsequent calculation of $d_{1}$ in the cross-section near the pin, $d_{2}$ as a multiplication of $d_{1}$, and finally $V_{f, c}(u)$ in each other cross-section, such as to determine the model parameters for both the assumed fiber volume fraction and fiber direction models.

The assumed fiber volume fraction $V_{f}(u, v)$ and fiber direction $\vec{f}_{l o c}(u, v)$ in a cross-sections are thus defined as follows for the generation of geometrical 
features:

$$
\begin{gathered}
V_{f}(u, v)= \begin{cases}V_{f, \text { lam }} & \text { for } d_{2} \leq v \\
V_{f, \text { lam }}+\left(\frac{v-d_{1}}{d_{2}-d_{1}}\right) \cdot\left(V_{f, c}(u)-V_{f, l a m}\right) & \text { for } d_{1} \leq v \leq d_{2} \\
V_{f, c}(u) & \text { for } v \leq d_{1}\end{cases} \\
\vec{f}_{\text {loc }}(u, v)= \begin{cases}\vec{f}_{\text {lam }} & \text { for } d_{2} \leq v \\
\vec{f}_{\text {lam }}+\left(\frac{v-d_{1}}{d_{2}-d_{1}}\right) \cdot\left(\vec{f}_{\text {bound }}(u)-\vec{f}_{\text {lam }}\right) & \text { for } d_{1} \leq v \leq d_{2} \\
\vec{f}_{\text {bound }}(u) & \text { for } v \leq d_{1}\end{cases}
\end{gathered}
$$

The fiber misalignemt $\theta_{m i s}(u, v)$ with respect to $\vec{f}_{\text {lam }}$ is often used to represent the local fiber direction in a lamina and is defined as the angle between $\vec{f}_{l o c}(u, v)$ and $\vec{f}_{\text {lam }}$.

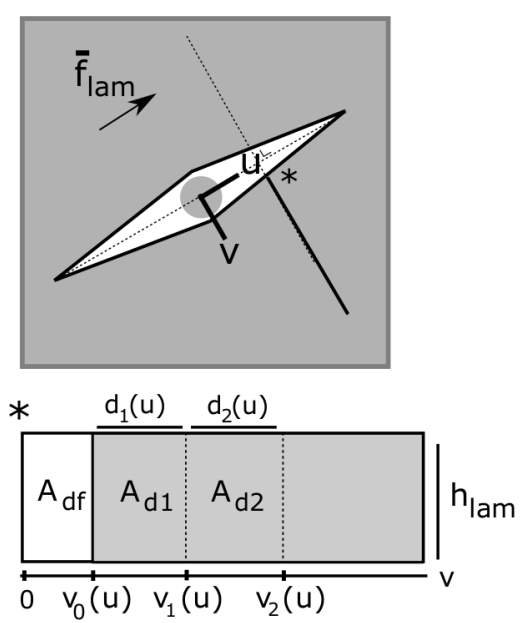

a)
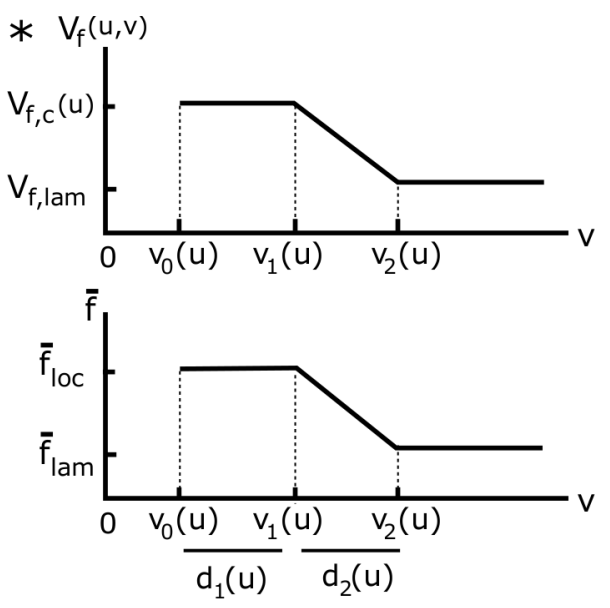

b)

Figure 7: Fiber-reinforced distorted zone model: (a) a cross-section indicating the model parameters, (b) the assumed fiber volume fraction and fiber direction distribution in the cross-section 


\section{Generated geometrical features}

\subsection{Shapes}

In Fig. 8 the computational approach proposed here is compared with the analytical approaches that uses orthorhombic and cosines functions [20, 29, for a pin diameter equal to $280 \mu \mathrm{m}$ and a resin-rich region length of $2000 \mu \mathrm{m}$ considering an opening distance of $4 \mu \mathrm{m}$. As can be observed in Fig. 8 , the model parameters used in the generation process resulted in a resin-rich region shape that has a smaller area and a larger maximum misalignment at the boundaries (Fig. 8b) compared to the analytical models. By increasing the number of straightening operations, and by allowing a larger opening distance, both the area and maximum misalignment can be satisfactorily recovered as from the functional representations. It can be concluded that the straightening operation, applied on locally deformed lines, is able to generate realistic shapes of resin-rich regions in comparison to the analytical modelling approach based on experimental observations [15, 16].

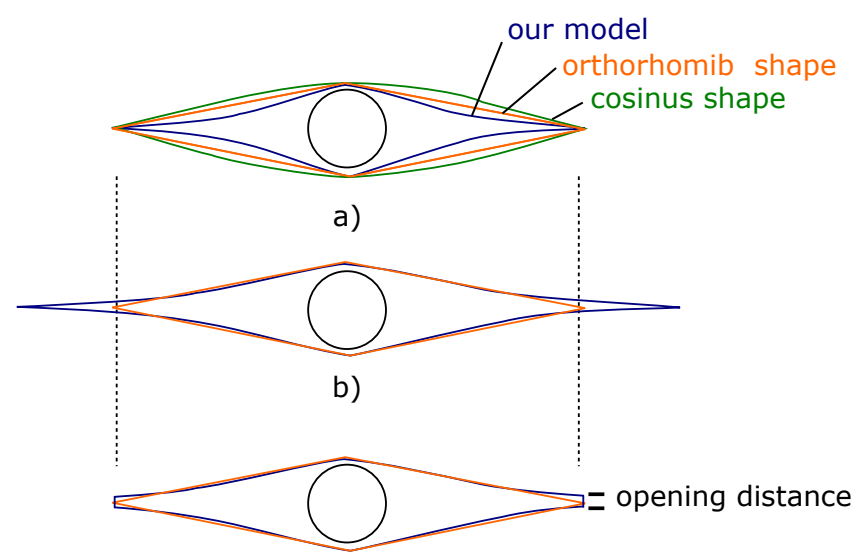

c)

Figure 8: Setting the tolerance opening distance and comparing the shaped of generated resin-rich regions with functional representations, hereby having used in the generation: (a) a tolerance opening distance of $4 \mu \mathrm{m}$, (b) an increased number of straightening operations, (c) and an increased tolerance opening distance.

An illustration of the possibility of the modelling approach to generate dif- 
ferent shapes of resin-rich regions and fiber-einforced distorted zones in a flexible manner for different pin parameters and stacking sequences is presented in Fig. 9. As can be seen, the modeling approach can account for the transition from eyelet-to-channel shapes for increasing pin content (Fig. 9a), the fiber weaving shape for irregularly positioned pins in a $0^{\circ}$-lamina (Fig. 9b), the symmetrical shapes for initially inclined pins and asymmetrical shapes for a pin inclined due to pin rotation in different lamina orientations (Fig. 9 ), and the possibility to control the out-of-plane position of the top boundary of a lamina that accounts for fiber crimp due to pin rotation (Fig. 9d).

\subsection{Dimensions}

The resin-rich region can be characterized by its width and length. The width is similar to the pin diameter, which is automatically obtained during generation. The length (which controls the addition of the eyelet-shape or channel-shape) can be regulated by the number of straightening operations.

The fiber-reinforced distorted zone can be characterized by its width, length, maximum fiber volume fraction, and out-of- plane fiber waviness. Its width is obtained automatically in the model by considering the amount of fibers contained in a cross-section and can be further regulated by adapting the distorted zone parameter $k$ (see Eq. 7). As can be seen in Fig. 10, the smallest distorted zone width is obtained for $k=0$, while for $k=20+$ larger distorted zone widths are obtained, representing a step-wise constant and linear varying fiber volume fraction or fiber misalignment profile respectively. Its length is equal to the resin-rich region length, as local distortions start emerging as soon as lines are moved locally. The maximum fiber volume fraction near the pin is a direct input parameter in the fiber-reinforced distorted zone model and was assumed equal to 0.900 which represents near the pin a fully compacted fiber-reinforced zone [32]. The out-of-plane fiber waviness can be controlled by constraining or un-constraining the positions of nodes on the bottom and top of lamina during generation which is directly related to presence of fiber crimping. 
a)
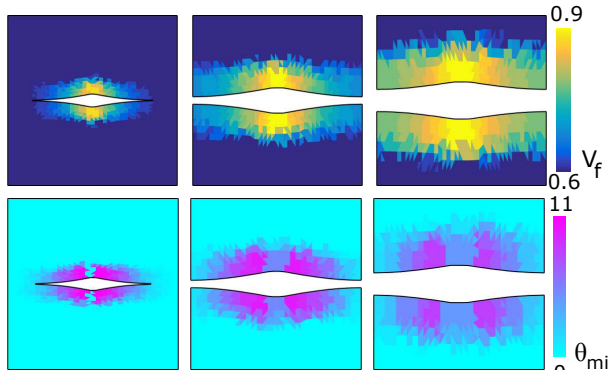

$0.5 \%$

$2.0 \%$

pin content

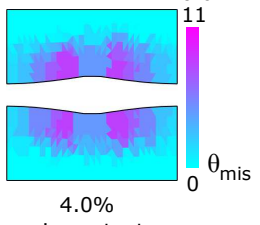

pin content

b)
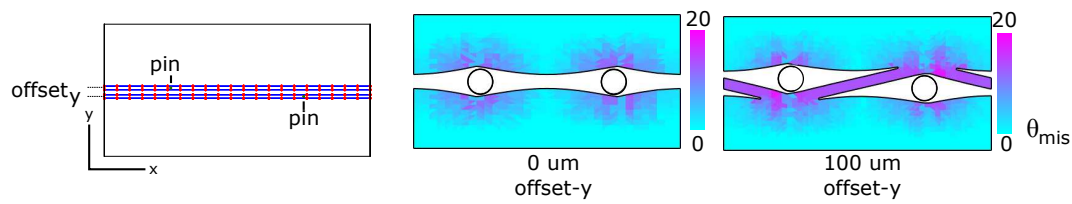

c)
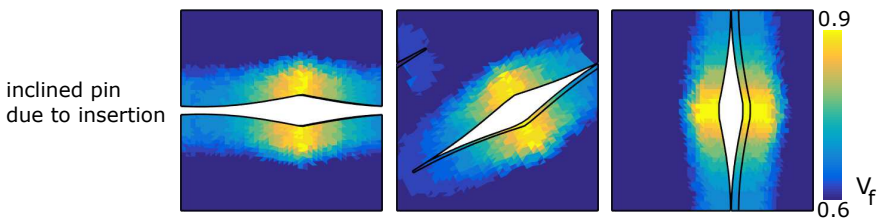

inclined pin due to rotation
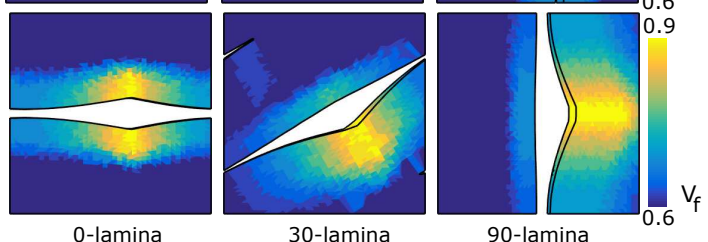

0-lamina

30-lamina

90-lamina

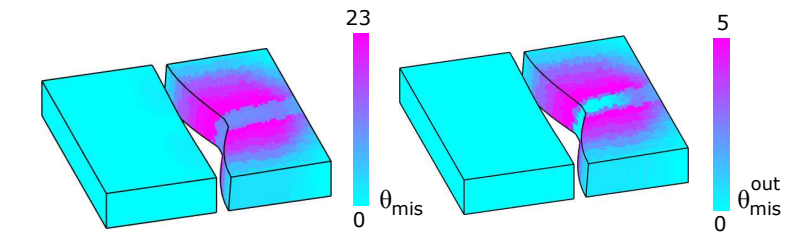

d)

Figure 9: Shapes of resin-rich regions and corresponding fiber volume fraction $V_{f}$ and fiber misalignment $\theta_{\text {mis }}$ distributions: (a) eyelet- and channel-shaped resin-rich regions corresponding to a small and large pin diameter, (b) fiber weaving for an irregular pin pattern, (c) symmetrical- and asymmetrical- resin-rich regions for an inclined pin configuration upon pin insertion or due to pin rotation respectively, (d) out-of-plane fiber misalignment $\theta_{\text {mis }}^{\text {out }}$ or fibercrimp due to pin rotation. 

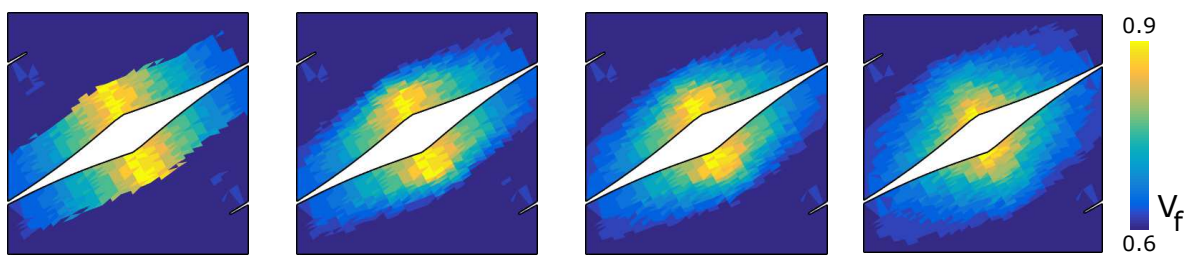

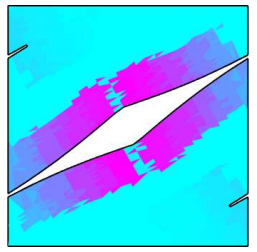

$\mathrm{k}=0$

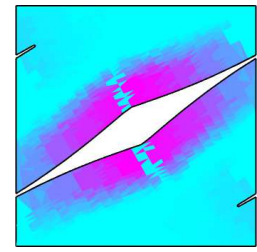

$k=1$

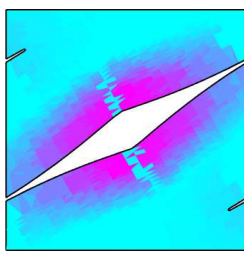

$\mathrm{k}=2$

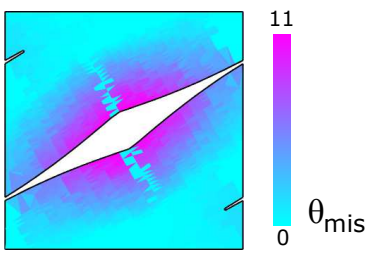

$k=20$

Figure 10: The effect of the distorted zone parameter $k$ on the fiber volume fraction $V_{f}$ and fiber misalignment distribution $\theta_{m i s}$ in lamina.

\section{Mechanical simulations}

Using the computationally built geometrical models developed here, finite element models can be generated automatically, hereby using the free 3D finite element mesh generation procedure GMSH [33 35]. A small matrix layer was inserted between the laminae to ease meshing (see Fig. 11). Linear elastic simulations with periodic boundary conditions are here performed for the purpose of analysing the effect of the detailed geometrical features associated with pinning. The effect of the stacking sequence on the reduction in global stiffness and the presence of stress concentrations that can explain early damage initiation for different pin contents [36, 37] are investigated on a lamina-level, by considering a single lamina with different fiber orientations and pin contents. The effect of the local fiber volume fraction as well as local fiber direction in a lamina, and the effect of the reinforcement distortions caused by pin rotation in a laminate are also investigated. Such effects are finally investigated at the scale of a laminate.

\subsection{Models}

Pinned lamina models are now generated with fibers in lamina oriented $0^{\circ}$, $30^{\circ}$ and $90^{\circ}$ from the tensile direction and a pin diameter of $280 \mu m$ (a horizontal 


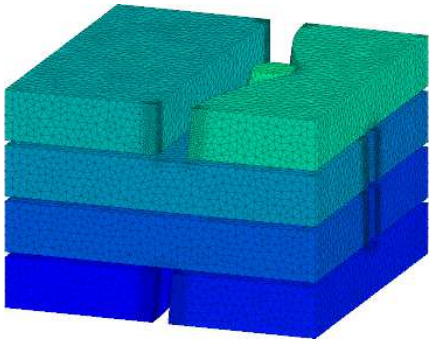

a)

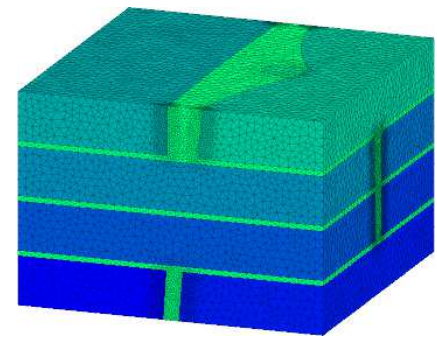

b)

Figure 11: Generated mesh: (a) without matrix, (b) with matrix.

tensile direction is assumed in the sequel). For each lamina configuration, the pin content, pin radius and fiber-reinforced region representation are varied as presented in Table 1

\begin{tabular}{llccc|cc}
\hline model & description & $V_{f}^{\max }$ & $\theta_{\text {mis }}^{\max }$ & $V_{\text {distzone }} / V_{\text {lam }}$ & pin content $(\%)$ & $R_{\text {pin }}($ um $)$ \\
\hline 1 & equivalent & 0.60 & 0 & 0 & - & - \\
2 & const $V_{f} /$ const $\vec{f}_{l o c}$ & 0.66 & 0 & 0.00 & 2 & 140 \\
3 & var $V_{f} /$ const $\vec{f}_{l o c}$ & 0.90 & 0 & 0.48 & 2 & 140 \\
4 & const $V_{f} / \operatorname{var} \vec{f}_{l o c}$ & 0.66 & 10 & 0.48 & 2 & 140 \\
5 & var $V_{f} / \operatorname{var} \vec{f}_{l o c}$ & 0.90 & 10 & 0.48 & 2 & 140 \\
6 & pin content 0.50 & 0.76 & 6 & 0.27 & 0.50 & 70 \\
7 & pin content 4 & 0.90 & 14 & 0.80 & 4 & 200 \\
\hline
\end{tabular}

Table 1: The lamina models with their geometrical characteristsics $\left(V_{\text {distzone }} / V_{\text {lam }}\right.$ indicates the percentage of waviness region inside the lamina).

The pin is considered to be vertical, or inclined due to pin rotation in the $\mathrm{x}$-direction, for which an inclined pin angle of 15 degrees is used. The model parameters that describe the position of the vertical and inclined pin, are given in Table 2,

For each configuration, equivalent lamina/laminate models are also generated. The same lamina dimensions are then considered in the equivalent configuration, hereby not accounting for laminate swelling that may be present upon 


\begin{tabular}{c|ccc}
\hline & $\alpha_{i}$ & $\alpha_{f}$ & $\alpha_{x y}$ \\
\hline vertical pin & 0 & 0 & 0 \\
inclined pin & 0 & 15 & 0 \\
\hline
\end{tabular}

Table 2: The pin parameters for the pinned laminate models.

pin insertion.

\subsection{Boundary conditions and mechanical analysis methodology}

The unit-cell model is constrained on its in-plane borders by periodic boundary conditions with the bottom and top surfaces of the unit-cell left free. Displacements are applied on the corner nodes to control the equivalent strain value [38. A strain of $0.5 \%$ is applied on the unit-cell, which would cause local failure to take place inside the distorted zone but not outside the distorted zone [36, 37.

The materials used in this analyses consists of carbon fibers, an epoxy matrix, and a metal pin, with properties as defined in Table 3. Material properties for the fiber-reinforced region, as function of local fiber volume fraction, are calculated using the analytical mechanical model of Chamis [39, using the previously defined properties; and are assigned afterwards in the local material system that is prescribed by the local fiber direction.

The mechanical models are analyzed in terms of global stiffness and local stresses. The global stiffness of a pinned laminate is normalized by the global stiffness of its equivalent unpinned laminate. Local stress levels are evaluated for the risk of local transverse and shear failure in the fiber-reinforced region, and matrix failure in the resin-rich region. Simple stress-based criteria are used 


\begin{tabular}{cccc|ccc}
\hline & carbon fiber & epoxy matrix & metal pin & & carbon fiber & epoxy matrix \\
\hline$E_{11}(G P a)$ & 231 & 3.45 & 200 & $X_{11, t}(M P a)$ & 3500 & 70 \\
$E_{22}(G P a)$ & 15 & - & - & $X_{11, c}(M P a)$ & 3000 & 130 \\
$E_{33}(G P a)$ & 15 & - & - & $X_{12}(M P a)$ & - & 57 \\
$v_{12}$ & 0.20 & 0.35 & 0.30 & & \\
$v_{13}$ & 0.20 & - & - & & \\
$v_{23}$ & 0.20 & - & - & & \\
$G_{12}(G P a)$ & 15 & 1.28 & 143 & & \\
$G_{13}(G P a)$ & 7 & - & - & & & \\
$G_{23}(G P a)$ & 7 & - & - & & & \\
\hline
\end{tabular}

Table 3: The stiffness and strength properties of carbon fiber (AS4 carbon [40]), epoxy matrix (5260 epoxy [40]) and pin.

to evaluate the potential damage initiation in the elements in each region:

$$
\begin{aligned}
& \text { transverse failure: } f_{22}=\frac{\sigma_{22}}{X_{22}\left(V_{f}\right)} \geq 1 \\
& \text { shear failure: } f_{12}=\frac{\sigma_{12}}{X_{12}\left(V_{f}\right)} \geq 1 \\
& \text { matrix failure: } f_{m}=\frac{\sigma_{p}}{X_{t}} \geq 1
\end{aligned}
$$

were $X_{t}$ is the matrix tensile strength, and $X_{22}$ and $X_{12}$ the local transverse strength and local shear strength in the fiber-reinforced region respectively. $\sigma_{p}$ is the maximum principle stress, with $f_{m}$ and $f_{i j}$ being defined as the local failure indicator for the different local damaging modes, and that indicate damage initiation when above one. The transverse and shear strength are calculated as a function of local fiber volume fraction and were obtained by the analytical Chamis model [39]. Stress concentrations are represented by a 97 -precentile of the local failure indicators:

$$
f_{i j}^{97 \%}=97 \operatorname{perc}\left(w_{k} . f_{i j}\right) \quad \text { with } \quad w_{k}=\frac{V_{\text {elem }, k}}{\Sigma V_{\text {elem }, k}}
$$

where $V_{\text {elem, },}$ is the volume of a finite element. Before analysis, a mesh refinement analysis was performed to analyze the effect of discretisation parameters on the global stiffness and local stresses. For a single lamina and with different fiber directions, a coarse and finer mesh were analyzed, with the finer mesh 
constructed by decreasing the mesh parameters belonging to the local mesh size fields. The results of the mesh comparison study are presented in Table 4. It can be seen that the results for the fine and coarse mesh are relatively similar with no large deviations, and as such it was concluded that the coarse mesh could be used in further mechanical analyses.

\begin{tabular}{l|ccc|ccc|ccc}
\hline & \multicolumn{3}{|c|}{$0^{\circ}$-lamina } & \multicolumn{3}{c|}{$30^{\circ}$-lamina } & \multicolumn{3}{c}{$90^{\circ}$-lamina } \\
& $E_{x}^{\text {norm }}$ & $f_{22}^{\%}$ & $f_{12}^{\%}$ & $E_{x}^{\text {norm }}$ & $f_{22}^{\%}$ & $f_{12}^{\%}$ & $E_{x}^{\text {norm }}$ & $f_{22}^{\%}$ & $f_{12}^{\%}$ \\
\hline fine mesh & 0.97 & 0.59 & 1.70 & 1.05 & 0.88 & 1.41 & 1.02 & 1.61 & 0.16 \\
coarse mesh & 0.97 & 0.59 & 1.60 & 1.05 & 0.90 & 1.43 & 1.02 & 1.62 & 0.16 \\
\hline
\end{tabular}

Table 4: The normalised stiffness and the 97-percentile of the transverse and shear cracking failure indicators of the pinned lamina which is meshed by two different meshs sizes.

\subsection{Results}

\subsubsection{Single lamina}

The global stiffness of pinned laminae with different fiber orientations and pin contents is presented in Table 5 . It can be seen that the $0^{\circ}$-lamina has a reduction in stiffness, while for the $30^{\circ}$ - and $90^{\circ}$-laminae an increase in stiffness is observed with the change in stiffness depending on the pin content. These characteristics can be explained by considering the analytical mechanical model which relates the local fiber misalignment with respect to a loading direction to the local stiffness (e.g. Piggott [41]) and by accounting for the presence of the pin. As illustrated in Fig. 12, a fiber misalignment in the distorted zone in a lamina for different lamina orientations can either decrease or increase the local stiffness, dependent on whether the fiber misalignments tends to make the fibers become more aligned or less aligned with the loading direction. For offaxis laminae, the presence of a pin acts as a stiffness enhancing medium. The combined effect of fiber misalignment and pin inclusion can either decrease or increase the global stiffness. These results can then help explaining the differences in reduction of global stiffness of pinned laminates for different stacking 
sequences and pin contents [11.

\begin{tabular}{c|ccc}
\hline lamina orientation & $0^{\circ}$ & $30^{\circ}$ & $90^{\circ}$ \\
\hline pin content $0.5 \%$ & 0.99 & 1.02 & 1.01 \\
pin content $2.0 \%$ & 0.97 & 1.05 & 1.02 \\
pin content $4.0 \%$ & 0.90 & 1.12 & 1.06 \\
\hline
\end{tabular}

Table 5: The stiffness of a pinned lamina for different pin content and orientations of the main lamina's fiber direction in the lamina.

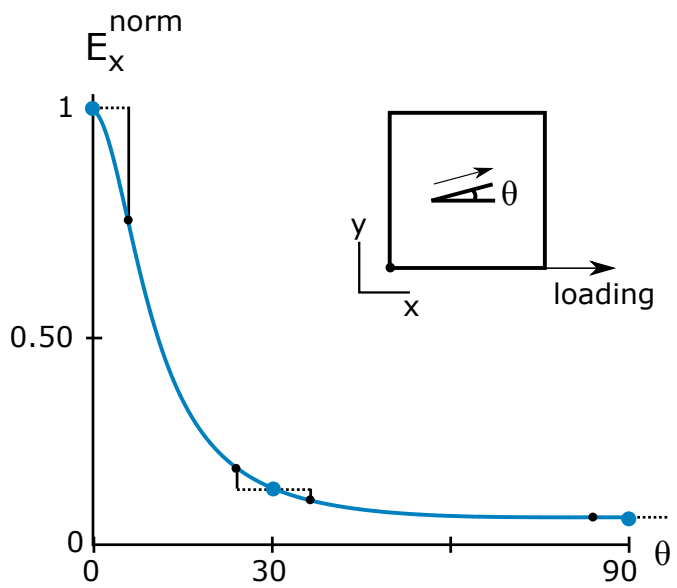

Figure 12: A graph illustrating the effect of fiber misalignment $\theta_{m i s}$ on the stiffness $E$ whereby the dashed lines show that the fiber misalignment $\theta_{\text {mis }}$ can either decrease or increase the stiffness (having used hereby the analytical model presented in for example 41]).

The local stresses are analyzed in terms of their location within the lamina, with the contributing effects of local fiber volume fraction and fiber direction on these values being assessed. From the contour plots of local failure indicators as presented in Fig. 13, it can be seen that the stress concentrations are taking place in the fiber-reinforced distorted zone and, that for the applied strain, local damage is initiated inside the distorted zone but not in the undistorted zone. The stress components that are causing these stress concentrations are either the shear stress for a $0^{\circ}$-lamina and a $30^{\circ}$-lamina, or the transverse stress for 
a $90^{\circ}$-lamina. The local failure indicator concentrations can be increased by a factor 2 or 3 with respect to the values in an equivalent unpinned lamina (see Table 6). This is further correlated with the pin content as depicted in Fig. 15 The effect of local fiber volume fraction and fiber direction on the local failure indicators is illustrated in Fig. 14 by plotting the results from the different models as described in Table1. It can be seen that the increaese of fiber volume fraction in the distorted region has the largest contribution to the local stress concentrations compared to fiber misalignment. These results confirm that, for pinned laminates, earlier damage initiation may indeed take place. The results also demonstrate the importance of a proper evaluation of the local fiber volume fraction for a relevant evaluation of local stress concentrations in computational models, and their potential in partly explaining the observed scatter in global strength [11].

\begin{tabular}{c|cc|cc|cc}
\hline & \multicolumn{2}{|c}{$0^{\circ}$-lamina } & \multicolumn{2}{c}{$30^{\circ}$-lamina } & \multicolumn{2}{c}{$90^{\circ}$-lamina } \\
& $f_{22}^{97 \%}$ & $f_{12}^{97 \%}$ & $f_{22}^{97 \%}$ & $f_{12}^{97 \%}$ & $f_{22}^{97 \%}$ & $f_{12}^{97 \%}$ \\
\hline equivalent & 0.00 & 0.00 & 0.34 & 0.63 & 0.62 & 0.00 \\
$\operatorname{var} V_{f} / \operatorname{var} \vec{f}_{l o c}$ & 0.59 & 1.60 & 0.90 & 1.43 & 1.62 & 0.16 \\
\hline
\end{tabular}

Table 6: The 97-percentile of the transverse and shear failure indicators in an equivalent and pinned lamina with different orientations of their main fiber direction.

\subsubsection{Laminate}

The global stiffness of the $\left[0^{\circ} / 90^{\circ} / 90^{\circ} / 0^{\circ}\right]$ laminate for both a vertical and inclined pin (see section 4.1) is presented in Table 7 for both $\mathrm{x}$ - and y-direction loading. It can be seen that a reduction in global stiffness is present for all cases and that the reduction is larger for the inclined pinned laminate when loaded transversally to the inclination direction. This enlarged reduction for $y$-loading (see Fig. 16a) can be explained by the larger fiber distortion region that are present in the main load-bearing lamina (90-lamina), while in the case of $\mathrm{x}$ loading, the main load-bearing lamina $0^{\circ}$-lamina) are only slightly distorted. These results indicate that the geometrical distortions due to pin rotations can 
a)
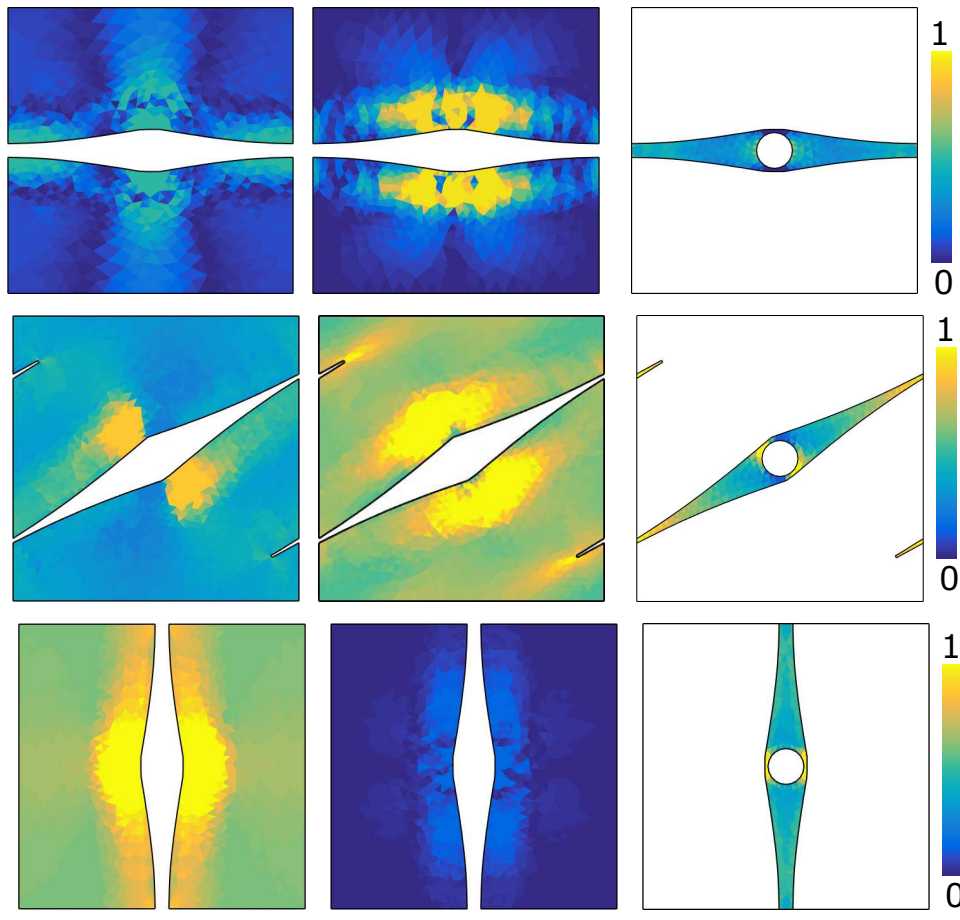

$f_{22}$
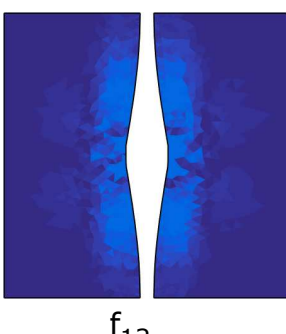

$f_{12}$
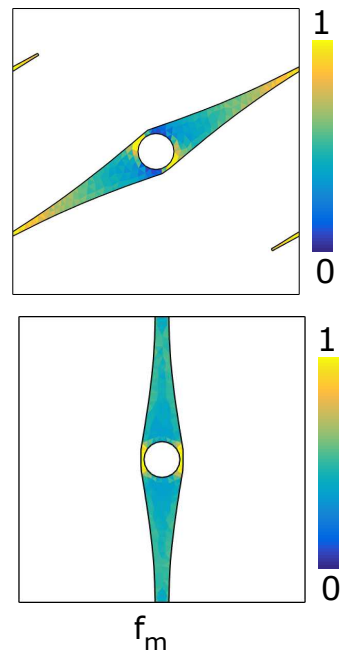

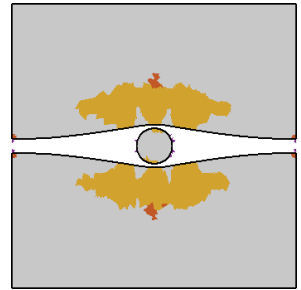

$\mathrm{f}_{22}>1$

$\mathrm{f}_{12}>1$

$\mathrm{f}_{\mathrm{m}}>1$

Figure 13: Transverse, shear and matrix cracking local failure indicator distributions for a lamina with fibers aligned in different directions: (a) $0^{\circ}$-lamina, (b) $30^{\circ}$-lamina, (c) $90^{\circ}$ lamina.
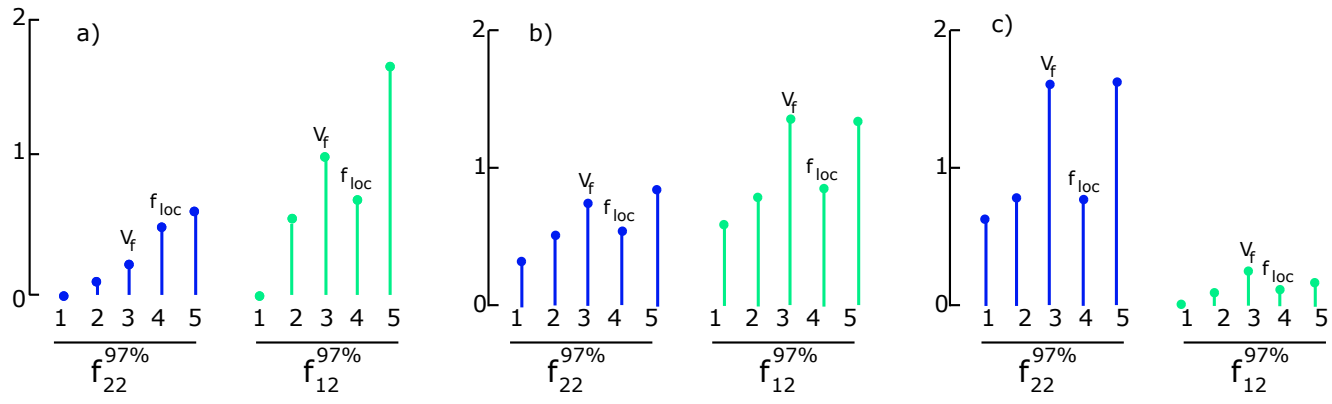

Figure 14: Graphs showing the role of fiber volume fraction $V_{f}$ and fiber misalignment $\theta_{\text {mis }}$ on the 97-percentile of the transverse and shear cracking local failure indicators for lamina with fibers aligned in different directions: (a) $0^{\circ}$-lamina, (b) $30^{\circ}$-lamina and (c) $90^{\circ}$-lamina. 

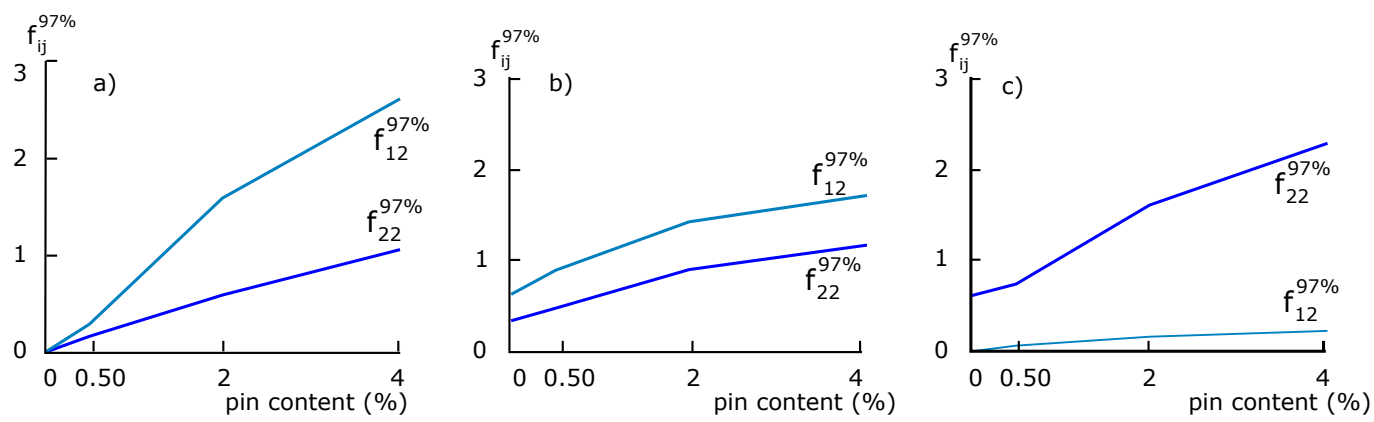

Figure 15: Graphs indicating the effect of pin content on the 97-percentile of the transverse and shear cracking local failure indicators in a lamina with fibers algined in different directions: (a) $0^{\circ}$-lamina, (b) $30^{\circ}$-lamina and (c) $90^{\circ}$.

affect the global stiffness, which can partly explain the scatter in global stiffness as observed in experimental results and need to be included in geometrical models.

Local stress distributions for values of $f_{i j}>1$ are illustrated by contour plots of the local failure indicators in Fig. 16b. It can be seen that the stress concentration are correlated with the fiber-reinforced distorted zone (see Fig. 16a) and that both transverse, shear, matrix damage initiation may be present. Mechanisms accounting for these local degradations should therefore be included for strength modelling of Z-pinned laminates.

\begin{tabular}{ccc}
\hline & $E_{x}^{\text {norm }}$ & $E_{y}^{\text {norm }}$ \\
\hline vertical pin & 0.98 & 0.98 \\
inclined pin & 0.98 & 0.84 \\
\hline
\end{tabular}

Table 7: The normalised stiffness of the pinned laminate models in both in-plane directions.

\subsection{Discussion}

The simulations result are now compared with experimental data presented in [11] that show the stiffness of unidirectional $\left[0^{\circ}\right]$, cross-ply $\left[0^{\circ} / 90^{\circ}\right]$ and quasiisotropic $\left[0^{\circ} / 45^{\circ} /-45^{\circ} / 90^{\circ}\right]$ pinned laminates (normalised by the stiffness of 

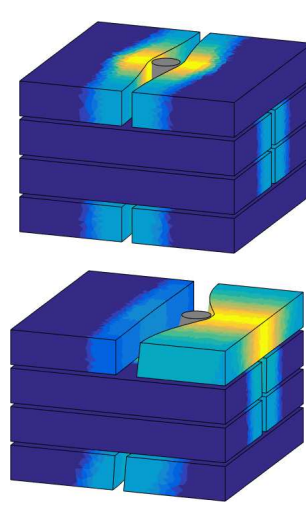

$\mathrm{Vf}$

0.6
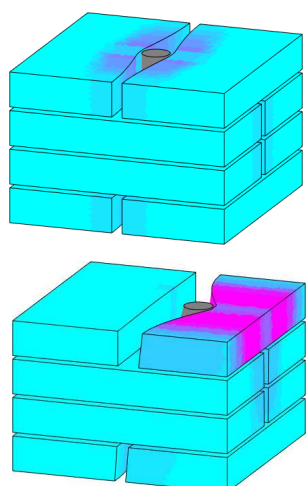

$\theta_{\text {mis }}$

0 20

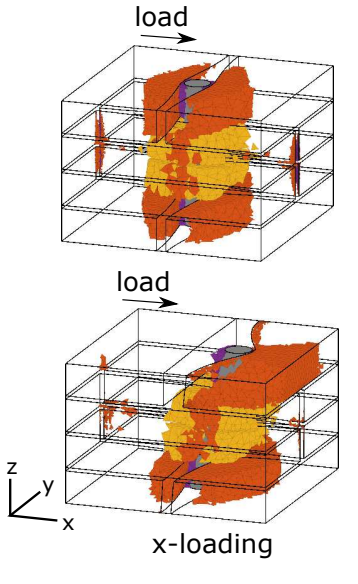

a)
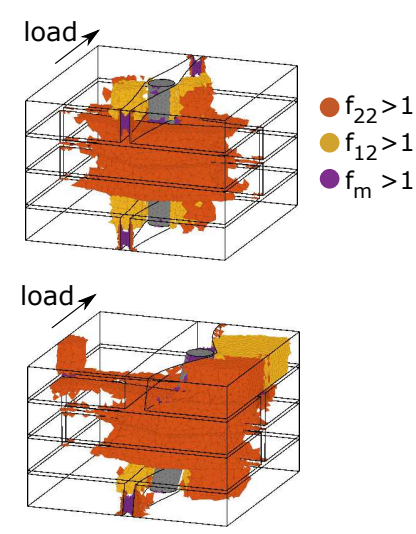

y-loading

b)

Figure 16: Geometrical and mechanical features of the vertical and inclined pinned laminate model: (a) fiber volume fraction $V_{f}$ and fiber misalignment $\theta_{\text {mis }}$ distributions, (b) the locally failed regions.

an equivalent unpinned laminate) for different pin contents. The normalised stiffness of the unidirectional, cross-ply and quasi-isotropic pinned laminates for pin contents of $2 \%$ is hereby approximately $0.87,0.94$ and 1.00 , respectively, while a linear trend is observed for increasing pin content.

\subsubsection{Lamina}

The experimental results indicate that distortions in the $0^{\circ}$-lamina reduce the stiffness to a larger degree compared to distortions in the $45^{\circ}$ - and $90^{\circ}$ lamina, and that the distortions in the $45^{\circ}$-lamina reduce the stiffness the least and may even increase the stiffness. Similar trends can be observed from the simulation results (presented in Table 5) that show that the distortions in the $0^{\circ}$-lamina, $90^{\circ}$-lamina and $30^{\circ}$-lamina are more negatively affecting the stiffness in a successive order respectively. The larger stiffness reduction in the experimental observations, compared to the simulation results, can be explained by not having considered lamina swelling, pin inclination, secondary geometrical features (broken fibers, fiber-crimp, interface cracking, etc.), which may further reduce the stiffness in the lamina modelling. 


\subsubsection{Laminate}

The normalised stiffness of the cross-ply laminates with a pin content of $2 \%$ from the experimental observations (0.94) and the simulations (being 0.98 and 0.84 for an inclined pin laminate models with pin inclination due to pin insertion and pin rotation respectively, see Table 7) can be compared. The difference between the physical and numerical model values can be explained by differences in geometrical features similar to as presented in section 4.4.1 including some further remarks on pin inclination angle and loading direction:

1. Physically, the pin inclination angle is characterised by a Gaussian distribution with a mean for pin diameters of $280 \mu \mathrm{m}$ which gradually evolves from $2^{\circ}$ on average after pin insertion to approximately $15^{\circ}$ on average after consolidation [15]. Numerically, the pin inclination angle was considered as $15^{\circ}$ and either obtained from pin insertion or from pin rotation (see Table 2). The considered $0^{\circ}$-value for the pin inclination angle upon pin insertion can then be considered as a worst case scenario whereby variations in pin inclination angles (which can be up to $20^{\circ}$ ) can further affect the results.

2. The loading direction with respect to the inclination direction can affect the stiffness (see Table 7), but experimental observations do not make a distinction between the in-plane direction and consequently average out the effect of different pin inclination directions. Averaging the simulation results (0.98 and 0.84 ) would then lead to a closer estimate compared the experimentally observed value 0.94 .

A parametric study including variations in pin inclination angle and direction, and laminate swelling, should then be performed to understand in a quantitative manner the differences between the experimental data and simulations results.

\section{Conclusion}

A computational approach was presented for a fully automated generation of mesoscopic geometrical unit-cell models of pinned laminates. The resin-rich re- 
gions are modelled by initial straight discretized lines that are gradually shaped using geometrical operations mimicking pin-insertion, pin rotation, and fiber deflections. The fiber-reinforced distorted zone is modelled in a post-processing stage in cross-sections perpendicular to the main fiber direction and on which a certain fiber volume fraction is assumed based on the preservation of the amount of fibers in the cross-section during generation. The shapes of the geometrical features are obtained computationally and in an automated way without the need to be set a priori as for analytical modelling approaches. The ability of the defined approach to obtain shapes computationally and to generate shapes of the main geometrical features belonging to a range of pin parameters and stacking sequences was also presented.

The geometrical models can be automatically transformed into FE mechanical models, based on which the effect of distortions on global stiffness and local stress distribution can be investigated. The following was observed:

1. The global stiffness of a pinned lamina can both be decreased and increased, depending on the main fiber direction with respect to the tensile direction.

2. The geometrical distortions caused by pin rotation can have a large effect on the global stiffness when loaded transversally to the inclination direction.

3. The fiber-reinforced distorted zone is acting as a stress concentration regions and both transverse, shear, and matrix damage initiation can be present at early strain values.

4. The fiber volume fraction is the main geometrical feature affecting the stress concentration. The results can help explaining the effect of the stacking sequence and the experimentally observed scatter on global stiffness and strength of Z-pinned laminates.

These results emphasize the importance of properly incorporating geometrical features of reinforcement distortions caused by pin rotations and variations 
in fiber volume fraction in mesoscopic models for Z-pinned laminates. These features have been only partly accounted for by past geometrical models [27 29] and have clearly been shown to affect the material properties of the laminates modelled here.

In future work, the presented computational approach for geometrical model generation will be adopted and extended to generate geometrical unit-cell models for stitched composites, in which geometrical features of local distortions of the reinforcement caused by the stitching yarn need to be accounted for.

\section{Acknowledgements}

The first author acknowledges the support of IWT under Grant No. IWTSB-678.

[1] D. D. Cartié, B. Cox, N. Fleck, Mechanisms of crack bridging by composite and metallic rods, Composites Part A: Applied Science and Manufacturing 35 (11) (2004) 1325-1336.

[2] B. Cox, Snubbing effects in the pullout of a fibrous rod from a laminate, Mechanics of Advanced Materials and Structures 12 (2) (2005) 85-98.

[3] H. Cui, Y. Li, S. Koussios, L. Zu, A. Beukers, Bridging micromechanisms of z-pin in mixed mode delamination, Composite Structures 93 (11) (2011) $2685-2695$.

[4] S. Kravchenko, O. Kravchenko, M. Wortmann, M. Pietrek, P. Horst, R. B. Pipes, Composite toughness enhancement with interlaminar reinforcement, Composites Part A: Applied Science and Manufacturing 54 (2013) 98-106.

[5] M. Yasaee, J. Lander, G. Allegri, S. Hallett, Experimental characterisation of mixed mode traction-displacement relationships for a single carbon composite z-pin, Composites Science and Technology 94 (2014) 123-131. 
[6] B. M'membe, S. Gannon, M. Yasaee, S. R. Hallett, I. K. Partridge, Mode ii delamination resistance of composites reinforced with inclined z-pins, Materials \& Design 94 (2016) 565-572.

[7] K. Rugg, B. Cox, R. Massabo, Mixed mode delamination of polymer composite laminates reinforced through the thickness by z-fibers, Composites Part A: applied science and manufacturing 33 (2) (2002) 177-190.

[8] I. K. Partridge, D. D. Cartié, Delamination resistant laminates by z-fiber@ pinning: Part i manufacture and fracture performance, Composites Part A: applied science and manufacturing 36 (1) (2005) 55-64.

[9] D. W. Johnson, S. A. Garrett, J. M. Hook, S. G. Moyers, Method of inserting z-axis reinforcing fibers into a composite laminate, uS Patent 6,645,333 (Nov. 11 2003).

[10] A. Mouritz, Review of z-pinned composite laminates, Composites Part A: applied science and manufacturing 38 (12) (2007) 2383-2397.

[11] A. Mouritz, B. Cox, A mechanistic interpretation of the comparative inplane mechanical properties of 3d woven, stitched and pinned composites, Composites Part A: Applied Science and Manufacturing 41 (6) (2010) 709728.

[12] A. Mouritz, Compression properties of z-pinned composite laminates, Composites Science and Technology 67 (15-16) (2007) 3110-3120.

[13] A. Mouritz, P. Chang, Tension fatigue of fibre-dominated and matrixdominated laminates reinforced with z-pins, International Journal of Fatigue 32 (4) (2010) 650-658.

[14] C. A. Steeves, N. A. Fleck, In-plane properties of composite laminates with through-thickness pin reinforcement, International Journal of Solids and Structures 43 (10) (2006) 3197-3212. 
[15] P. Chang, A. Mouritz, B. Cox, Properties and failure mechanisms of zpinned laminates in monotonic and cyclic tension, Composites Part A: Applied Science and Manufacturing 37 (10) (2006) 1501-1513.

[16] P. Chang, Mechanical properties and failure mechanisms of z-pinned composites, Ph.D. thesis, RMIT University (2006).

[17] C. A. Steeves, N. A. Fleck, In-plane properties of cfrp laminates containing throughthickness reinforcing rods (z-pins), ICCM-12, Paris.

[18] R. Sevenois, D. Garoz, F. Gilabert, S. Spronk, S. Fonteyn, M. Heyndrickx, L. Pyl, D. Van Hemelrijck, J. Degrieck, W. Van Paepegem, Avoiding interpenetrations and the importance of nesting in analytic geometry construction for representative unit cells of woven composite laminates, Composites Science and Technology 136 (2016) 119-132.

[19] S. V. Lomov, D. S. Ivanov, I. Verpoest, M. Zako, T. Kurashiki, H. Nakai, S. Hirosawa, Meso-fe modelling of textile composites: Road map, data flow and algorithms, Composites Science and Technology 67 (9) (2007) 18701891.

[20] H. Heß, Y. Roth, N. Himmel, Elastic constants estimation of stitched ncf cfrp laminates based on a finite element unit-cell model, Composites Science and Technology 67 (6) (2007) 1081-1095.

[21] S. Green, M. Matveev, A. Long, D. Ivanov, S. Hallett, Mechanical modelling of 3d woven composites considering realistic unit cell geometry, Composite Structures 118 (2014) 284-293.

[22] D. S. Ivanov, F. Baudry, B. Van Den Broucke, S. V. Lomov, H. Xie, I. Verpoest, Failure analysis of triaxial braided composite, Composites Science and Technology 69 (9) (2009) 1372-1380.

[23] A. Melro, P. Camanho, F. A. Pires, S. Pinho, Numerical simulation of the non-linear deformation of 5-harness satin weaves, Computational Materials Science 61 (2012) 116-126. 
[24] A. Doitrand, C. Fagiano, F.-H. Leroy, A. Mavel, M. Hirsekorn, On the influence of fabric layer shifts on the strain distributions in a multi-layer woven composite, Composite Structures 145 (2016) 15-25.

[25] S. Daggumati, W. Van Paepegem, J. Degrieck, J. Xu, S. V. Lomov, I. Verpoest, Local damage in a 5-harness satin weave composite under static tension: Part ii-meso-fe modelling, Composites Science and Technology 70 (13) (2010) 1934-1941.

[26] B. Piezel, B. Mercatoris, W. Trabelsi, L. Laiarinandrasana, A. Thionnet, T. Massart, Bending effect on the risk for delamination at the reinforcement/matrix interface of $3 \mathrm{~d}$ woven fabric composite using a shell-like rve, Composite structures 94 (8) (2012) 2343-2357.

[27] L. Dickinson, G. Farley, M. Hinders, Prediction of effective threedimensional elastic constants of translaminar reinforced composites, Journal of composite materials 33 (11) (1999) 1002-1029.

[28] M. Grassi, X. Zhang, M. Meo, Prediction of stiffness and stresses in z-fibre reinforced composite laminates, Composites Part A: applied science and manufacturing 33 (12) (2002) 1653-1664.

[29] B. Zhang, G. Allegri, M. Yasaee, S. Hallett, Micro-mechanical finite element analysis of z-pins under mixed-mode loading, Composites Part A: Applied Science and Manufacturing 78 (2015) 424-435.

[30] B. Sonon, T. J. Massart, A level-set based representative volume element generator and xfem simulations for textile and 3d-reinforced composites, Materials 6 (12) (2013) 5568-5592.

[31] B. Wintiba, B. Sonon, K. E. M. Kamel, T. J. Massart, An automated procedure for the generation and conformal discretization of $3 \mathrm{~d}$ woven composites rves, Composite Structures 180 (2017) 955-971.

[32] S. V. Lomov, E. Belov, T. Bischoff, S. B. Ghosh, T. T. Chi, I. Verpoest, Carbon composites based on multiaxial multiply stitched preforms. part 1. 
geometry of the preform, Composites Part A: Applied science and manufacturing 33 (9) (2002) 1171-1183.

[33] C. Geuzaine, J.-F. Remacle, Gmsh: A 3-d finite element mesh generator with built-in pre-and post-processing facilities, International journal for numerical methods in engineering 79 (11) (2009) 1309-1331.

[34] J.-F. Remacle, C. Geuzaine, G. Compère, E. Marchandise, High-quality surface remeshing using harmonic maps, International Journal for Numerical Methods in Engineering 83 (4) (2010) 403-425.

[35] E. Marchandise, C. C. de Wiart, W. Vos, C. Geuzaine, J.-F. Remacle, Highquality surface remeshing using harmonic maps - part ii: Surfaces with high genus and of large aspect ratio, International Journal for Numerical Methods in Engineering 86 (11) (2011) 1303-1321.

[36] V. Koissin, J. Kustermans, S. V. Lomov, I. Verpoest, B. Van Den Broucke, V. Witzel, Structurally stitched ncf preforms: quasi-static response, Composites science and technology 69 (15) (2009) 2701-2710.

[37] D. Mikhaluk, T. Truong, A. Borovkov, S. V. Lomov, I. Verpoest, Experimental observations and finite element modelling of damage initiation and evolution in carbon/epoxy non-crimp fabric composites, Engineering Fracture Mechanics 75 (9) (2008) 2751-2766.

[38] V. V. Kouznetsova, Computational homogenization for the multi-scale analysis of multi-phase materials, Ph.D. thesis, Technische Universiteit Eindhoven (2002).

[39] C. C. Chamis, Mechanics of composite materials: past, present, and future, Journal of Composites, Technology and Research 11 (1) (1989) 3-14.

[40] A. Kaddour, M. Hinton, P. Smith, S. Li, Mechanical properties and details of composite laminates for the test cases used in the third world-wide failure exercise, Journal of Composite Materials 47 (20-21) (2013) 2427-2442. 
[41] P. MR., Load bearing fiber composites, oxford: Pergamon press, 1980. p. 74. 